\title{
An investigation of flavor encapsulation comprising of regenerated cellulose as core and carboxymethyl cellulose as wall
}

\author{
Ming Ma $\cdot$ Lanlan Tan $\cdot$ Ya Dai $\cdot$ Jinping Zhou
}

Received: 27 February 2013/Accepted: 17 June 2013/Published online: 28 June 2013

(c) The Author(s) 2013. This article is published with open access at Springerlink.com

\begin{abstract}
The potential application of flavor encapsulation comprising regenerated porous cellulose particles (RPC) as core and carboxymethyl cellulose (CMC) as wall was investigated using L-menthol as a model flavor. RPC was prepared by sol-gel transition method and characterized by Fourier-transform infrared (FTIR) spectra, scanning electron microscopy (SEM) and micromeritics instruments. The results revealed that the RPC showed the particle size about $300-500 \mu \mathrm{m}$ as well as the specific surface area about $8.7 \mathrm{~m}^{2} / \mathrm{g}$. Based on high adsorptive capability, RPC could encapsulate menthol well and offer high encapsulation yield. The menthol content of RPC-menthol complexes (RPCM) was measured by gas chromatogram (GC) and showed the maximum content of about $12 \%$ menthol with the encapsulation efficiency of $70 \%$. Besides, RPC provided comparable flavor retention as microcrystalline cellulose during storage under relative humidity of $80 \%$ at $25{ }^{\circ} \mathrm{C}$. CMC was then used to modify RPCM and the construction of RPCM-CMC was confirmed by FTIR and SEM. The studies showed that CMC wall on RPCM surface had no influence on the menthol content and encapsulation efficiency in encapsulation process, but provided a significant increase in menthol retention during storage depending on the content of CMC in RPCM-CMC. Moreover, the stability test at various temperatures showed that both RPCM and RPCM-CMC
\end{abstract}

\footnotetext{
M. Ma $(\bowtie) \cdot$ J. Zhou

Department of Chemistry, Wuhan University, Wuhan 430072 ,

People's Republic of China

e-mail: spacemaming@163.com

M. Ma · L. Tan · Y. Dai

Technical Research Center, China Tobacco Chuanyu Industrial Co., Chengdu 610065, People's Republic of China
}

were stable at room temperature and released flavor at temperatures common in food processing. It was concluded that RPC and CMC modification shows great potential as flavor encapsulant.

Keywords Regenerated cellulose particles · Flavor encapsulation · Flavor retention · Menthol

\section{Introduction}

Flavor plays an important role in food quality and influences consumers' satisfaction and food consumption. It has attracted significant attention on improving flavor retention due to the instability of volatile flavors in the presence of air, light, moisture or high temperature [13]. To protect the aroma compounds from degradation and limit their loss, it is beneficial to encapsulate the flavor prior to use.

Generally, an encapsulation matrix for flavor should have good solubility in water, emulsifying properties, drying properties and low viscosity at high solid content [4]. The materials meeting the above requirements could encapsulate flavor by spray drying, extrusion or other processes [5-7]. Cyclodextrin is one of the most common encapsulation matrixes and represents one of the simplest encapsulant systems. Cyclodextrin could form inclusion complexes with different flavors dependent on their hydrophobicity as well as molecular size and geometry [8-10]. The cyclodextrin inclusion complexes have been reported to have high encapsulation yield and long retention time [11-13]. Unfortunately, under the legislation in Europe, the use of $\beta$-cyclodextrin for encapsulation of flavor substances is only permitted to a certain extent, and for other cyclodextrins, hardly at all. 
Other excipients for encapsulation are also widely investigated, and good flavor retention has been reported $[14,15]$. Some of these excipients, in particular starch, maltodextrins, glucose and sucrose, are used by body for energy as well. However, the excess consumption of energy in food has been proved to be problematic and it is increasingly leading to obesity and to the associated concomitant diseases such as diabetes, cardio-circulatory disorders and muscular-skeletal and locomote system complaints. A high and ever-increasing proportion of the population, especially in the highly developed industrialized countries, has been suffering from the consequences of the excess consumption of energy. Therefore, the introduction of these substances should be controlled as for as possible.

Cellulose, the most abundant and renewable polysaccharide on earth, has been wildly used in the chemical and biological industries for its safe, biocompatible, hydrophilic and biodegradable natures [16-18]. It is noted that cellulose also has a neutral inherent taste as well as a regulatory effect on digestion. For all the stated advantages, cellulose is very suitable as flavor additive. However, cellulose is hard to dissolve in aqueous solutions due to the presence of strong inter- and intra-molecular hydrogen bonds and considerable van der Waals forces, limiting its application in flavor industry. Therefore, most studies and applications in flavor encapsulation about cellulose have been focused on its derivatives. Luo and Popplewell [19] employed a matrix containing hydroxyethyl cellulose (HEC) to encapsulate flavor or fragrance materials as food products and laundry applications. Roberts et al. [20] studied the effect of viscosity and thickener type (sucrose, guar gum, and carboxymethyl cellulose) on dynamic flavor release and found that the increase of viscosity and thickener of CMC resulted in lower release in flavor. Sansukcharearnpon et al. [21] prepared a polymer-blend of ethylcellulose (EC), hydroxypropyl methylcellulose (HPMC) and poly (vinyl alcohol) to encapsulate flavors, and found that menthol shows the slowest release. Recently, a flavoring substance based on cellulose was produced and provided a novel approach for flavor encapsulation [22]. In the flavoring substance, flavor was absorbed by cellulose and embedded in voids located between cellulose chains. Although a high-yield encapsulation was obtained, the retention of flavor in the flavoring substance was still limited due to the adsorptive capability of cellulose. In this work, the regenerated porous cellulose particles (RPC) with high adsorptive capability were prepared via a green path, and RPC's potential application in flavor encapsulation was investigated by taking menthol, one of the most popular food flavors as model. Moreover, $\mathrm{CMC}$ was used as the coating layer of RPC to improve the retention of flavors.

\section{Experimental}

\section{Materials}

Cellulose (cotton linter pulp) was provided by Hubei Chemical Fiber Group (Xiangfan, China). Its viscosityaverage molecular weight $\left(\mathrm{M}_{\eta}\right)$ was determined to be $8.1 \times 10^{4} \mathrm{~g} / \mathrm{mol}$. Carboxymethyl cellulose (CMC) with viscosity of $800-1,200 \mathrm{mPa} . \mathrm{s}$ was obtained from Sinopharm Chemical Reagent Co. (Shanghai, China). Microcrystalline cellulose (MCC) was purchased from Tianli Pharmacy Materials Company (Qufu, China). L-Menthol was obtained from Beijing Chemical Co. (Beijing, China). Other chemical reagents of analytical grade were purchased from China, and were used without further purification.

Preparation of regenerated porous cellulose particles

The RPC particles were prepared according to the previous method [23]. A solution with $\mathrm{NaOH} / \mathrm{urea} / \mathrm{H}_{2} \mathrm{O}(7: 12: 81$ by weight) was cooled to $-12{ }^{\circ} \mathrm{C} .16 \mathrm{~g}$ cellulose was immediately dispersed into the solvent system $(400 \mathrm{~mL})$ under vigorous stirring for $3 \mathrm{~min}$ to obtain a transparent cellulose solution. $300 \mathrm{~mL}$ sherwood oil and $10 \mathrm{~g}$ span 80 were added into a reactor and stirred at $800 \mathrm{rpm}$ for $20 \mathrm{~min}$, and then $400 \mathrm{~mL}$ cellulose solution was dropped into the reactor at room temperature. After stirring for $2 \mathrm{~h}$, the dilute hydrochloric acid (10\%) was added until $\mathrm{pH} 7$ to form RPC particles. The obtained particles were collected and washed with de-ionized water to remove the residual sherwood oil and span 80 . After drying at $80{ }^{\circ} \mathrm{C}$, the dried RPC for menthol encapsulation was obtained.

\section{Encapsulation of menthol}

The dried RPC particles ( $10 \mathrm{~g})$ were added to a threenecked flask with mechanical agitation. Menthol was dissolved in ethanol and the solution was added to RPC particles drop by drop under stirring. After adding menthol solution, stirring was still performed for $30 \mathrm{~min}$. Then, the flask was sealed and left for $5 \mathrm{~h}$. Dry air with temperature of $20^{\circ} \mathrm{C}$ was passed into the flask through one open neck and drawn off via another open neck to remove ethanol and the excess menthol. The obtained RPC-containing menthol particles were coded as RPC-menthol complex (RPCM).

The flavor encapsulant comprising RPC as core and $\mathrm{CMC}$ as wall was then prepared based on RPCM particles. Before RPCM was dried, $0.5 \%$ CMC aqueous solution was sprayed onto the surface of the particles, followed by dry air passed at $20{ }^{\circ} \mathrm{C}$ through the flask to remove the solvents and the excess menthol. Therefore, RPCM particles coated with CMC were prepared and coded as RPCM- 
CMC. The content of CMC was defined as the ratio of $\mathrm{CMC}$ added to the encapsulant.

$\mathrm{CMC}$ content $=\frac{\text { Total } \mathrm{CMC} \text { added }}{\text { Total encapsulant }} \times 100 \%$

The encapsulation efficiency (EE) was defined as the ratio of menthol in the dry encapsulation matrix to that menthol added.

Encapsulation efficiency (\%)

$=\frac{\text { Total menthol in encapsulation matrix }}{\text { Total menthol added }}$

The content of menthol was defined as the ratio of menthol in the dry encapsulation matrix to the dry encapsulation matrix.

Menthol content

$=\frac{\text { Total menthol in encapsulation matrix }}{\text { dry encaspulation matrix }} \times 100 \%$

The total menthol in encapsulation matrix was determined by gas chromatography (GC). In detail, $0.5 \mathrm{~g}$ of encapsulated samples was placed in the flask containing $20 \mathrm{~mL}$ ethanol. After ultrasonic at $40{ }^{\circ} \mathrm{C}$ for $30 \mathrm{~min}$, the extraction solution was collected and the menthol content in extraction solution was quantified by GC.

Microcrystalline cellulose-menthol particles were prepared by a similar way to RPCM, and named as MCCM.

\section{Menthol retention during storage}

Retention of menthol during storage was tested by incubation of the encapsulated samples under controlled relative humidity (RH) of $80 \%$ at $25^{\circ} \mathrm{C}$. RPCM and RPCM$\mathrm{CMC}$ were spread on watch glass with the thickness of $0.5 \mathrm{~cm}$. Menthol at different time intervals was determined by GC. MCCM was used as comparison.

The following setup was used to determine the retention at various temperatures. $50 \mathrm{mg}$ complex was placed in $20 \mathrm{~mL}$ phosphate buffer of $\mathrm{pH} 7.2(0.1 \mathrm{~mol} / \mathrm{L})$ in a $10 \mathrm{~mL}$ glass vial sealed by a screw cap covered with an aluminum foil, and incubated at 25,50 or $80^{\circ} \mathrm{C}$, respectively. After incubation for $4 \mathrm{~h}$, the samples were cooled and centrifuged $\left(2,000 \mathrm{rpm}, 2 \mathrm{~min}\right.$, and $\left.4{ }^{\circ} \mathrm{C}\right)$. The buffer solution was transferred into a separation funnel, and the menthol in the buffer solution was extracted by dichloromethane and quantified by GC.

\section{Characterization}

Fourier-transform infrared (FTIR) spectra were recorded on an FTIR spectrometer (model 1600, Perkin-Elmer Co.). Specific surface area was determined by nitrogen adsorption with Micromeritics instruments (Model ASAP 2020,
USA). The particle size and the surface morphology of encapsulant were characterized by Hitachi Table top Microscope TM-1000 (Germany).

Gas chromatography analysis was performed on an Agilent 5890 gas chromatograph (USA), using HP-5 fused silica capillary column $(30 \mathrm{~m} \times 0.25 \mathrm{~mm}$ with $0.25 \mu \mathrm{m}$ film thickness). The temperature programing for menthol detection was as follows: the temperature ramped at $10{ }^{\circ} \mathrm{C} /$ min from 50 to $100{ }^{\circ} \mathrm{C}$ and maintained for $1 \mathrm{~min}$; and then ramped at $5{ }^{\circ} \mathrm{C} / \mathrm{min}$ to $250{ }^{\circ} \mathrm{C}$ and maintained for $5 \mathrm{~min}$. The GC analysis conditions were as follows: the nitrogen carrier gas flow rate was $1.0 \mathrm{~mL} / \mathrm{min}$, hydrogen flow to the detector was $25 \mathrm{~mL} / \mathrm{min}$, air flow was $400 \mathrm{~mL} / \mathrm{min}$ and the flow of nitrogen makeup gas was $45 \mathrm{~mL} / \mathrm{min}$. Injection temperature was $250{ }^{\circ} \mathrm{C}$ and detection temperature was $250{ }^{\circ} \mathrm{C}$. The peaks were identified using HP GC ChemStation software. Standards for the aroma were used to identify the peaks.

\section{Results and discussion}

\section{Structure and morphology}

The porous regenerated cellulose particles were prepared using the sol-gel transition (SGT) method at room temperature. In our study, there was no evaporation of any chemical agents during dissolution of cellulose in $\mathrm{NaOH} /$ urea aqueous at low temperature, and the preparation method for RPC was simple and safe. Therefore, this is introduced as a "green" process. Moreover, the materials in preparation were all non-toxic and easily removed, ensuring the safety of the obtained RPC particles as flavor encapsulation matrix.

Figure 1 shows the SEM images of MCC, RPC and RPC-CMC. MCC showed the particle size between 100 and $200 \mu \mathrm{m}$, and the regularity of the crystal determined that MCC had smooth surface without porous structure. In contrast, RPC particles showed irregular spherical structure with the size of about $300-500 \mu \mathrm{m}$, and porous structure was observed on the particle surface. The pore formation was as a result of the $\mathrm{H}_{2} \mathrm{O}$ induced-phase separation during the sol-gel process, where the solvent-rich regions contributed to the pore formation [24]. The specific surface area of RPC was found $8.7 \mathrm{~m}^{2} / \mathrm{g}$ with an average pore diameter of $320 \mathrm{~nm}$, according to Brunauer-Emmett-Teller analysis. These results demonstrated that RPC might have more potential for encapsulating flavors than MCC. As CMC coating, the surface of RPC-CMC became smooth, and a thin film surrounding RPC was observed, indicating the formation of CMC wall.

Figure 2 shows the IR spectra of the RPC, RPCM and RPCM-CMC. In comparison with RPC, RPCM presented 

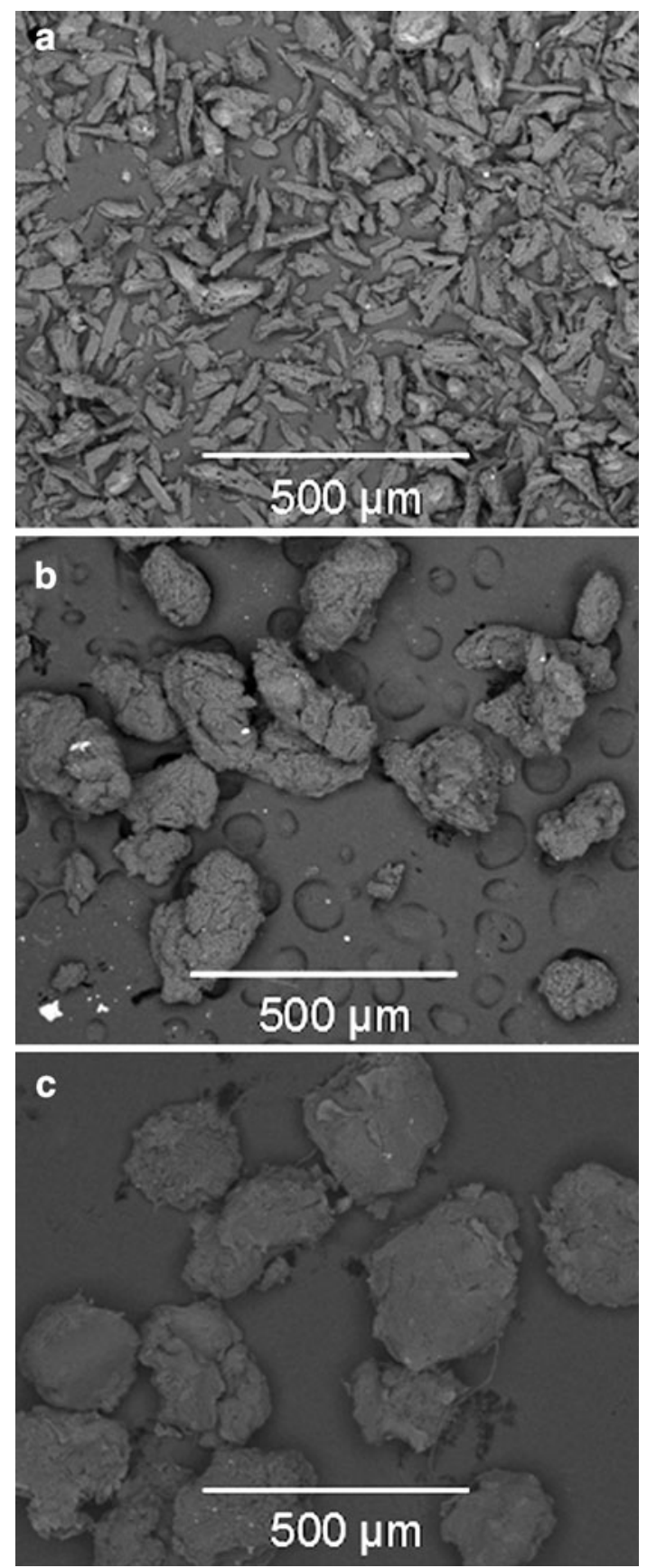

Fig. 1 SEM images of: a MCC, b RPC and c RPC-CMC

new peaks at $2,800-3,000 \mathrm{~cm}^{-1}$ and $1,026-1,047 \mathrm{~cm}^{-1}$ which were ascribed to the stretching vibrations of $\mathrm{C}-\mathrm{H}$ and $\mathrm{C}-\mathrm{O}$ in menthol, implying that menthol was successfully incorporated into RPC. For CMC-coated RPCM surface, the characteristic peaks of menthol at $2,800-3,000 \mathrm{~cm}^{-1}$ and $1,026-1,047 \mathrm{~cm}^{-1}$ were still maintained intact. In addition, the absorptions of $-\mathrm{OH}$ at $3,200-3,500 \mathrm{~cm}^{-1}$ and $1,640 \mathrm{~cm}^{-1}$ were greatly enhanced, which were probably due to the high hygroscopicity of CMC.

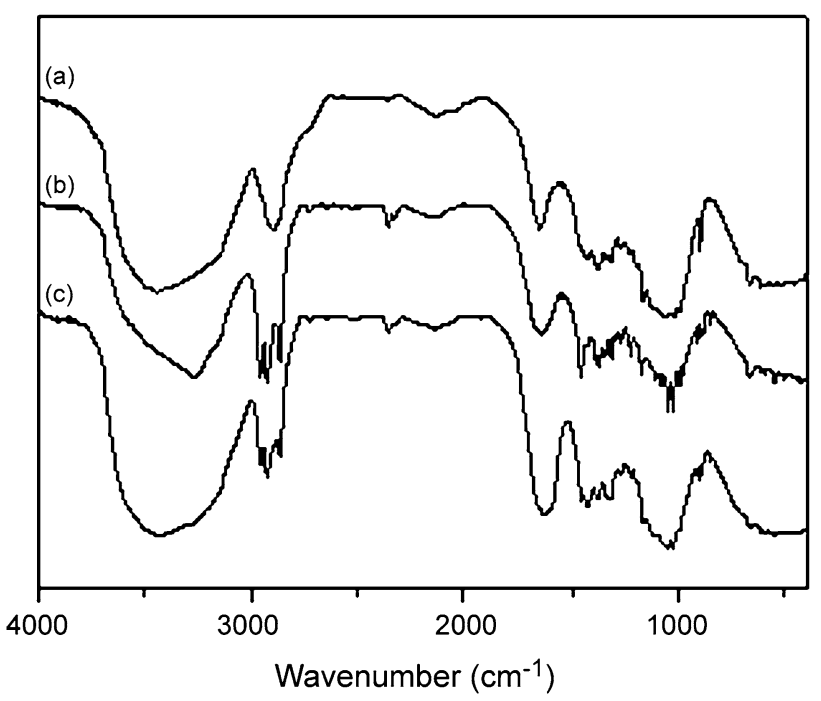

Fig. 2 FTIR spectra of encapsulant: a RPC, b RPCM and c RPCM$\mathrm{CMC}$

Menthol encapsulation

As it is well known, methanol is hard to dissolve in water, and its water solubility is about $1.3 \mathrm{~g} / \mathrm{L}$. The poor water solubility of methanol may impair the encapsulation process. As reported, several organic solvents instead of water were used for flavor encapsulation [25]. Here, ethanol, which has been considered as a good solvent for most flavors, was chosen as solvent in our study for its high dissolving capacity to methanol and easy elimination.

To evaluate the capability of RPC to encapsulate menthol, a series of concentrations of menthol solution was mixed at constant volume with $10 \mathrm{~g}$ RPC. Figure 3 reveals that RPC is capable to encapsulate menthol well. As the menthol concentration increased from 0.10 to $0.30 \mathrm{~g} / \mathrm{mL}$, its content in RPC ranged from 4.8 to $12.5 \%$. A further increase of menthol concentration could not significantly increase the content of menthol in RPCM, indicating that the maximum menthol retention in RPCM was reached. The menthol encapsulation efficiency (EE) in Fig. 4 shows different trends in menthol content. Before reaching the maximum content of menthol, the EE showed a little fall as the menthol concentration increased from 0.10 to $0.25 \mathrm{~g} /$ $\mathrm{mL}$. When the content of menthol reached the maximum, the EE falled severly, which might be due to the removal of excess menthol in encapsulation process. These results indicated that menthol concentration in encapsulation process is carefully controlled to avoid its wastage. For MCCM, the maximum menthol content was only about $5.9 \%$ at menthol concentration of $0.15 \mathrm{~g} / \mathrm{mL}$ with EE of $64.5 \%$, much less than RPCM, suggesting RPC was a more efficient encapsulant for menthol. 


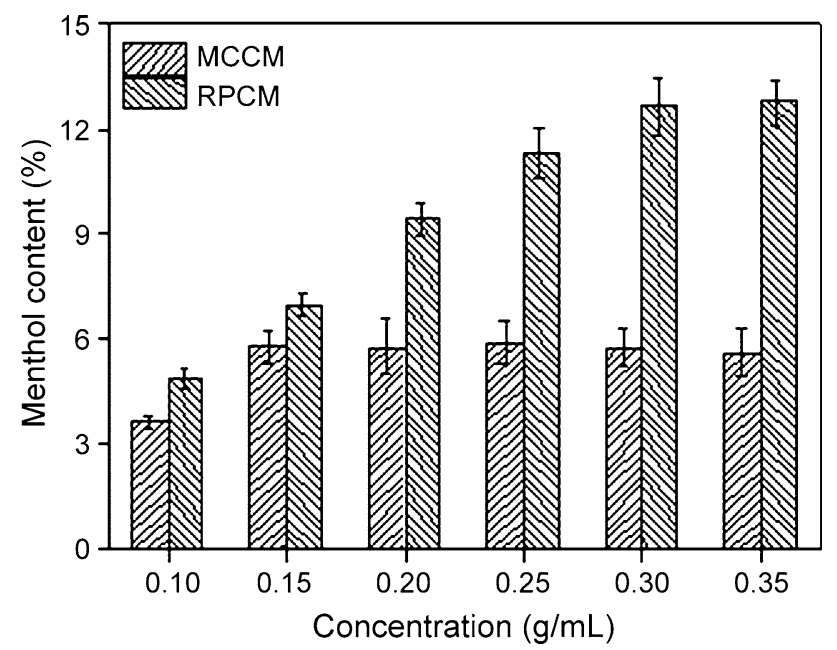

Fig. 3 The content of methanol in RPCM and MCCM. Values were expressed as mean values $\pm \mathrm{SD}$ of three independent tests

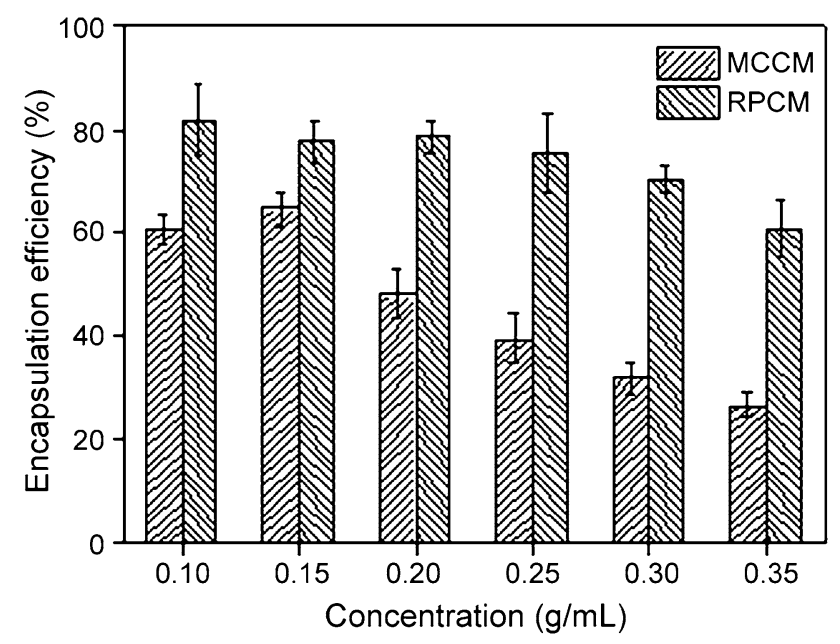

Fig. 4 Encapsulation efficiency of menthol in RPCM and MCCM. Values were expressed as mean values \pm SD of three independent experiments

According to the above results, $0.25 \mathrm{~g} / \mathrm{mL}$ menthol solution was considered best for preparing RPCM-CMC. In detail, $50 \mathrm{~mL}$ menthol solution was added to $100 \mathrm{~g}$ RPC under stirring, and then $\mathrm{CMC}$ solution was sprayed on the surface of RPCM as the outside shell. After removal of ethanol and water, the flavor particles comprising of RPCM as core and CMC as shell were obtained (Fig. 1c).

As a good wall material, CMC could hold and seal core materials entirely during the encapsulation process and storage [26]. As shown in Fig. 5, the content of methanol in RPCM-CMC showed no significant change in comparison with that in RPCM when various CMCs were involved. The corresponding EE (Fig. 6) was in good agreement with the content of menthol (Fig. 5). These results indicated that the CMC wall was suitable to serve as good wall material for RPCM.

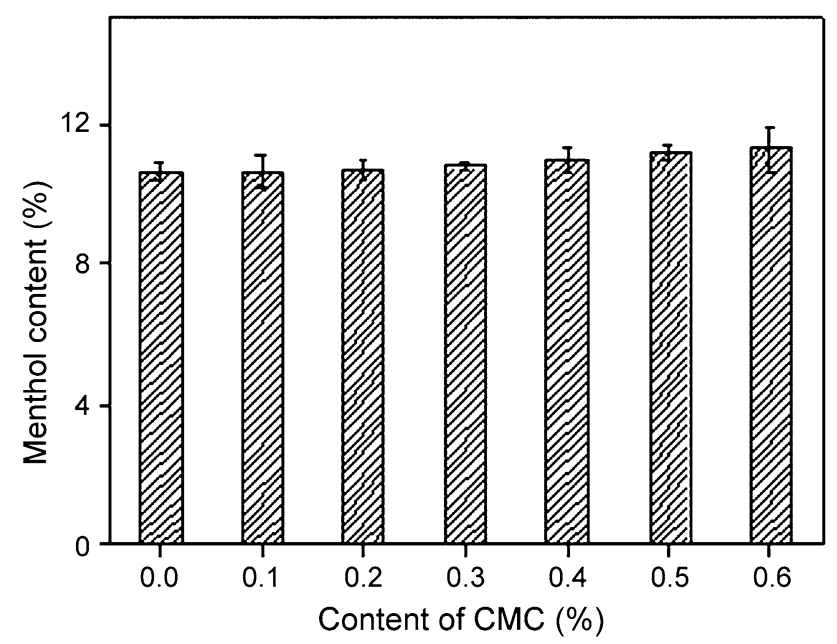

Fig. 5 The content of menthol in RPCM-CMC. Values were expressed as mean values \pm SD of three independent experiments

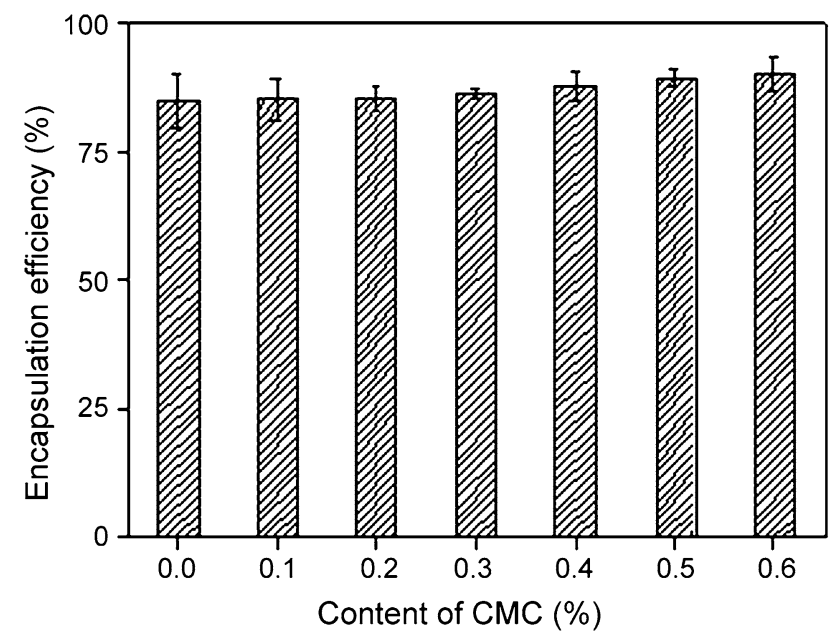

Fig. 6 Encapsulation efficiency of menthol in RPCM-CMC. Values were expressed as mean values $\pm \mathrm{SD}$ of three independent experiments

\section{Menthol retention}

It is important for flavor matrix to be stable during storage. To evaluate the potential of RPC and the platform as a flavor encapsulant, the retention of menthol in RPCM and RPCM-CMC was compared to that in MCCM under controlled $\mathrm{RH} 80 \%$ at $25{ }^{\circ} \mathrm{C}$. The results are shown in Fig. 7 as the percentage of total menthol recorded in comparison with the initial total menthol. As shown in Fig. 7, no menthol was found after incubation for 10 days, and this result was in agreement with the previous report [27]. The menthol retention in MCCM and RPCM showed a timedependent pattern. In the case of MCCM, about $73 \%$ menthol was left after 10 days of incubation, but more than $60 \%$ menthol was released after incubation for 60 days, implying that MCC had low capability to keep menthol. 


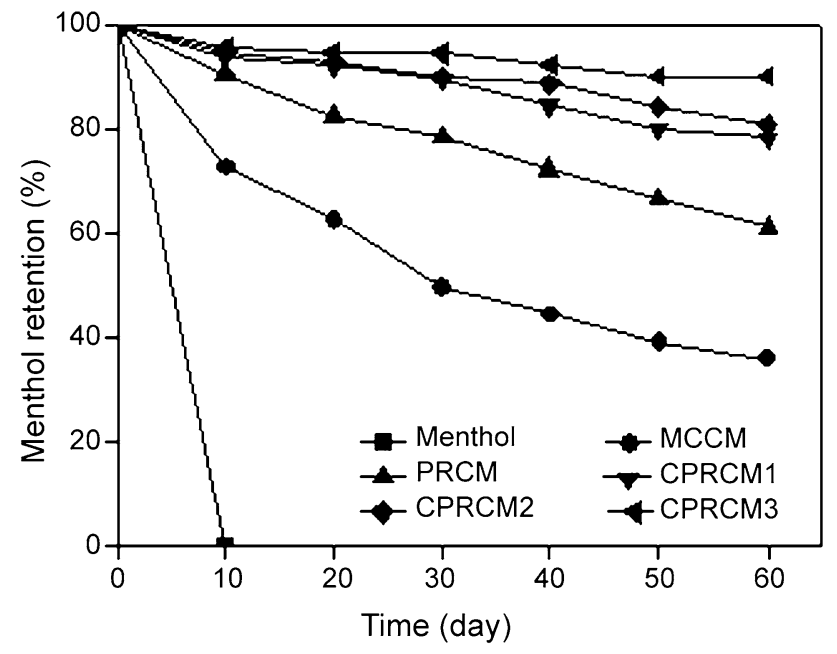

Fig. 7 Menthol retention in the complex during incubation at $25{ }^{\circ} \mathrm{C}$ RH $80 \%$ for 60 days as compared to microcrystalline cellulose. Menthol released as the percentage of total menthol recorded compared to the initial total menthol. Values are mean for $n=3$. The contents of CMC in RPCM-CMC were: RPCM-CMC1, $0.2 \%$; RPCM-CMC2, $0.4 \%$; and RPCM-CMC3, $0.6 \%$

For RPCM, the menthol retention was greatly improved in comparison with MCCM. After 10 days of incubation, about $90 \%$ menthol was left in RPCM, which was over $15 \%$ increase compared to MCCM. After incubation for 60 days, about $60 \%$ menthol was found in RPCM, over $25 \%$ greater than that of MCCM. The improvement of menthol retention in RPCM demonstrated that RPC could be a better flavor encapsulant than MCC.

When CMC was coated on the surface of RPCM, the menthol retention was further improved and showed a significant dependence on the CMC content. After 10 days of incubation, the series of RPCM-CMC showed little difference in menthol retention, but about $5 \%$ increase could be observed compared to RPCM. After 40 days, as the CMC content increased, the percentages of menthol left in the complexes were 84, 89, and $93 \%$ for RPCM-CMC1, RPCM-CMC2 and RPCM-CMC3, respectively. After incubation for 60 days, more menthol was released from the complexes, but there was at least $75 \%$ menthol remained in RPCM-CMC1, which was about 40 and $15 \%$ higher in comparison with MCCM and RPCM, respectively. With the increase in the CMC content, about 82 and $90 \%$ menthol retentions were left in RPCM-CMC2 and RPCM-CMC3, respectively. These results indicated that CMC wall on RPCM surface successfully reduces the loss of menthol during its storage.

Retention at various temperatures was also examined to determine the thermal stability of RPCM and RPCMCMC3. The results in Fig. 8 show the percentage of released menthol from these two complexes with the change of temperature. As temperature rose from 25 to

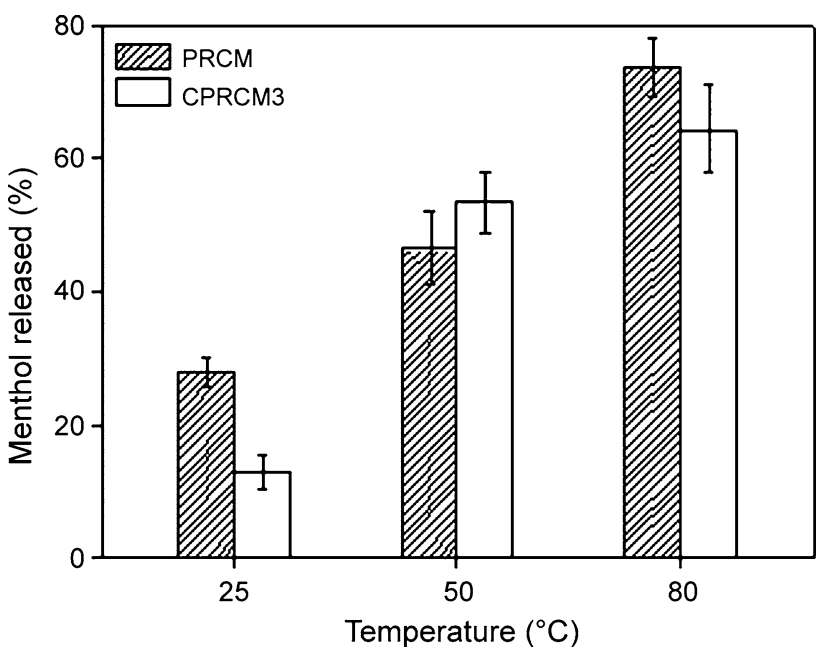

Fig. 8 Menthol release from the complex after incubation at 25, 50 or $80{ }^{\circ} \mathrm{C}$ for $4 \mathrm{~h}$. Menthol release is pronounced as percentage from total menthol. Values are mean \pm standard deviation, for $n=9$

$80{ }^{\circ} \mathrm{C}$, the percentage of released menthol increased from 27 to $73 \%$ for RPCM, and from 12 to $64 \%$ for RPCMCMC3, respectively. The fact indicated that both RPCM and RPCM-CMC3 were much more stable at room temperature than that at higher temperature. That might be because at higher temperature a temperature effect developed on aroma retention, leading to higher menthol release from the complex. This property of RPCM and RPCMCMC3 decided that both of them could be suitable as species in food processing.

\section{Conclusion}

Regenerated porous cellulose particles with a diameter of $300-500 \mu \mathrm{m}$ were prepared by SGT and were evaluated for their potential application in flavor encapsulation using menthol as model. Studies showed that the maximum menthol content in RPCM was about $12 \%$ with the encapsulation efficiency of $70 \%$. CMC film modifying RPCM had no significant influence on menthol content and encapsulation efficiency. The study of the menthol retention during storage indicated that RPCM and RPCMCMC showed longer retention time and the increase of CMC content in RPCM-CMC further prolonged the retention time during storage. Besides, the stability of menthol at different temperatures indicated that both RPCM and RPCM-CMC were suitable as species in food processing.

Open Access This article is distributed under the terms of the Creative Commons Attribution License which permits any use, distribution, and reproduction in any medium, provided the original author(s) and the source are credited. 


\section{References}

1. Manojlovic V, Rajic N, Djonlagic J, Obradovic B, Nedovic V, Bugarski B (2008) Application of electrostatic extrusion-flavour encapsulation and controlled release. Sensors 8:1488-1496

2. Bhandari BR, D'Arcy BR, Thi Bich LL (1998) Lemon oil to betacyclodextrin ratio effect on the inclusion efficiency of betacyclodextrin and the retention of oil volatiles in the complex. J Agric Food Chem 46:1494-1499

3. Peter S, Given J (2009) Encapsulation of flavors in emulsions for beverages. Curr Opin Colloid Interf 14:43-47

4. Trubiano PE, Lacourse NL (1988) In: Risch SJ, Reineccius GA (eds) Flavor encapsulation. American Chemical Society, Washington, pp 45-54

5. Dong ZJ, Ma Y, Hayat K, Jia C, Xia S, Zhang XM (2011) Morphology and release profile of microcapsules encapsulating peppermint oil by complex coacervation. J Food Eng 104:455-460

6. Yuliani S, Torley PJ, D'Arcy B, Nicholson T, Bhandari B (2006) Extrusion of mixtures of starch and D-limonene encapsulated with beta-cyclodextrin: flavour retention and physical properties. Food Res Int 39:318-331

7. Kant A, Linforth RT, Hort J, Aylor AJT (2004) Effect of betacyclodextrin on aroma release and flavor perception. J Agric Food Chem 52:2028-2035

8. Piel G, Dive G, Evrard B, Van Hees T, de Hassonville SH, Delattre L (2001) Molecular modeling study of beta- and gammacyclodextrin complexes with miconazole. Eur J Pharm Sci 13:271-279

9. Faucci MT, Melani F, Mura P (2002) Computer-aided molecular modeling techniques for predicting the stability of drug-cyclodextrin inclusion complexes in aqueous solutions. Chem Phys Lett 358:383-390

10. Marques HMC (2010) A review on cyclodextrin encapsulation of essential oils and volatiles. Flavour Frag J 25:313-326

11. Reineccius TA, Reineccius GA, Peppard TL (2002) Encapsulation of flavors using cyclodextrins: comparison of flavor retention in alpha, beta, and gamma types. J Food Sci 67:3271-3279

12. Reineccius TA, Reineccius GA, Peppard TL (2004) Utilization of $\beta$-cyclodextrin for improved flavor retention in thermally processed foods. J Food Sci 69:FCT58-FCT62

13. Madene A, Jacquot M, Scher J, Desobry S (2006) Flavour encapsulation and controlled release-a review. Int $\mathrm{J}$ Food Sci Tech 41:1-21
14. Paramita V, Furuta T, Yoshii H (2012) High-oil-load encapsulation of medium-chain triglycerides and D-limonene mixture in modified starch by spray drying. J Food Sci 77:E38-E44

15. Mourtzinos I, Kalogeropoulos N, Papadakis SE, Konstantinou K, Karathanos VT (2008) Encapsulation of nutraceutical monoterpenes in $\beta$-cyclodextrin and modified starch. J Food Sci 73:S89S94

16. Rusli H, Gandasasmita S, Amran MB (2013) Cellulose acetatesilica fume membrane: characterization and application for separation of starch and maltose. Iran Polym J 22:335-340

17. Fareghi AR, Moghaddam PN, Entezami AA, Avval ME (2013) Modification of hydrophilic cellulose fibers by monolayer growth of polystyrene chains using ATRP. Iran Polym J 22:361-367

18. Bagheri M, Shateri S (2012) Thermosensitive nanosized micelles from cholesteryl-modified hydroxypropyl cellulose as a novel carrier of hydrophobic drugs. Iran Polym J 21:365-373

19. Luo WC, Popplewell LM (2003) Hydroxyethyl cellulose encapsulation material. Patent No: US 2003/0077378 A1

20. Roberts DD, Elmore S, Langley KR, Bakker J (1996) Effects of sucrose, guar gum, and carboxymethyl cellulose on the release of volatile flavor compounds under dynamic conditions. J Agric Food Chem 44:1321-1326

21. Sansukcharearnpon A, Wanichwecharungruang S, Leepipatpaiboon N, Kerdcharoen T, Arayachukeat S (2010) High loading fragrance encapsulation based on a polymer-blend: preparation and release behavior. Int J Pharm 391:267-273

22. Siegel S (2008) Flavoring-substances included cellulose. Patent No: US 20100129516

23. Luo XG, Zhang L (2010) Creation of regenerated cellulose microspheres with diameter ranging from micron to millimeter for chromatography applications. J Chromatogr A 28:5922-5929

24. Cai J, Zhang LN (2006) Unique gelation behavior of cellulose in $\mathrm{NaOH} /$ urea aqueous solution. Biomacromolecules 7:183-189

25. Eineccius TR, Eineccius GR, Peppard TL (2005) The effect of solvent interactions on $\alpha$-, $\beta$-, and $\gamma$-cyclodextrin/flavor molecular inclusion complexes. J Agric Food Chem 53:388-392

26. Habi Mat Dian NL, Sudin N, Affandi Yusoff MS (1996) Characteristics of microencapsulated palm-based oil as affected by type of wall material. J Sci Food Agric 70:422-426

27. Ades H, Kesselman E, Ungar Y, Shimoni E (2012) Complexation with starch for encapsulation and controlled release of menthone and menthol. LWT Food Sci Technol 45:277-288 